\title{
Entanglement properties of quantum spin chains
}

\author{
Stein Olav Skrøvseth* \\ Department of Physics, Norwegian University of Science and Technology, N-7491 Trondheim, Norway
}

(Dated: 1 March 2006)

\begin{abstract}
We investigate the entanglement properties of a finite size $1+1$ dimensional Ising spin chain, and show how these properties scale and can be utilized to reconstruct the ground state wave function. Even at the critical point, few terms in a Schmidt decomposition contribute to the exact ground state, and to physical properties such as the entropy. Nevertheless the entanglement here is prominent due to the lower-lying states in the Schmidt decomposition.

PACS numbers: 03.67.Mn, 03.65.Ud, 75.10.Pq, 05.70.Jk
\end{abstract}

\section{INTRODUCTION}

The EPR argument [1] and the Bell inequalities 2] were, albeit with almost 30 years in between, acknowledgments that quantum theory exhibits the strange correlations known as entanglement. However, only in the latest few years has it been known that entanglement is a resource that can be utilized in quantum computing, [3] and is thus a central subject in the continually expanding field of quantum information theory. Furthermore, entanglement has been shown to be a fundamental feature in quantum phase transitions, something that has spawned a whole new field of research [4, 5, 6, 7, 8, 9, 10, 11, 12, 17]. This article will focus on entanglement in both quantum critical and non critical systems, the structure of entanglement in condensed matter systems being at its most complex in critical systems.

The determination of the ground state in a quantum system such as 1D spin chains is highly complex, but the last years have seen the development of techniques such as DMRG 13, 14 and the recent entanglement renormalization 15 which solve the problem efficiently. In both cases the entanglement in the problem seems to be the key to the methods' success over traditional renormalization schemes. Moreover, for a pure state, any long range correlations require the existence of entanglement in the state.

A quantum system with wave function $|\Psi\rangle$ in a Hilbert space $\mathcal{H}$, which is partitioned into two subspaces $\mathcal{H}_{\mathscr{A}} \otimes$ $\mathcal{H}_{\mathscr{B}}$, can be written as a Schmidt decomposition

$$
|\Psi\rangle=\sum_{n=1}^{\chi} \sqrt{\lambda_{n}}\left|\psi_{n}^{(\mathscr{A})}\right\rangle \otimes\left|\psi_{n}^{(\mathscr{B})}\right\rangle,
$$

where $\left|\psi_{n}^{(\mathscr{A})}\right\rangle \in \mathcal{H}_{\mathscr{A}}$ and vice versa. The coefficients $\lambda_{n}$ are real, positive c-numbers and the states are mutually orthogonal, $\left\langle\psi_{n}^{\mathscr{A}} \mid \psi_{m}^{\mathscr{A}}\right\rangle=\delta_{n m}$. The upper limit $\chi$, the Schmidt number, is a brute measure of the entanglement between the two subsystems, running from one (no entanglement), maximally reaching the dimensionality of the

${ }^{*}$ Electronic address: stein.skrovseth@phys.ntnu.no smallest of the two Hilbert spaces. The reduced density matrix of (say) system $\mathscr{A}$ can then be written

$$
\rho^{(\mathscr{A})}=\operatorname{Tr}_{\mathscr{B}}|\Psi\rangle\left\langle\Psi\left|=\sum_{n=0}^{\chi} \lambda_{n}\right| \psi_{n}^{(\mathscr{A})}\right\rangle\left\langle\psi_{n}^{(\mathscr{A})}\right|
$$

and equivalently for $\mathscr{B}$. Hence, knowing the Schmidt decomposition of the wave function is equivalent to knowing the basis in which the reduced density matrix is diagonal, modulo phases. We will in this article show that the effective Schmidt number $\chi_{\text {eff }}$ is much smaller than the possible maximum, and thus the number of terms contributing significantly to the wave function is surprisingly small. Remarkably, this also is true at highly entangled points in the phase space, such as at criticality.

The entropy of the wave function $|\Psi\rangle$ can be measured as the entropy of the subsystem,

$$
S_{\mathscr{A}}=-\operatorname{Tr} \rho^{(\mathscr{A})} \log _{2} \rho^{(\mathscr{A})}=-\sum_{n} \lambda_{n} \log _{2} \lambda_{n},
$$

increasing from zero if the two subspaces are entangled. Hence, computing the eigenvalues $\lambda_{n}$ of the reduced density matrix is vital to the understanding of entangled states.

\section{ISING MODEL}

The quantum Ising chain in external field $h$ in $1+1$ dimension is a good benchmark tool for the analysis of entanglement since its properties are extremely well known. The model is defined by the Hamiltonian

$$
H_{\text {Ising }}=-\sum_{n=1}^{N}\left(\sigma_{n}^{x} \sigma_{n+1}^{x}+h \sigma_{n}^{z}\right),
$$

on $N$ lattice sites. We operate with open boundary conditions (OBC), since this will ease the formalities when partitioning the system. This will weaken the phase transition in the sense that the conformal symmetry is broken compared to periodic boundary conditions (PBC) [16], but the phase transition in the thermodynamic limit will prevail. Also, Calabrese and 
Cardy have found that one can compute conformal signatures in the $\mathrm{OBC}$ case provided the boundary conditions are conformal [17]. The model has a phase transition at $h=1$ between the product state $\left|\Psi_{\infty}\right\rangle=$ $|\uparrow \uparrow \ldots \uparrow\rangle$ for $h>1$ and the Schrödinger cat state $\left|\Psi_{0}\right\rangle=\frac{1}{\sqrt{2}}(|\rightarrow \rightarrow \cdots \rightarrow\rangle+|\leftarrow \leftarrow \cdots \leftarrow\rangle)$ when $h<1$. Here $|\uparrow\rangle$ is the eigenstate of $\sigma^{z}$ with eigenvalue 1 , and $|\leftrightarrows\rangle$ are eigenstates of $\sigma^{x}$ with eigenvalues \pm 1 . Note that the transition is between unit entropy in the low field limit and zero in the high field limit.

Doing a Jordan-Wigner transform as sketched in Refs. [8, 18], we can map the model onto a string of non interacting fermions, and thus compute the eigenvalues for the Ising model (and a larger class of models) in what resembles the thermodynamic limit, typically a few hundred particles. In essence, we define the $N$ fermionic operators

$$
\hat{a}_{n}=\frac{1}{2}\left(\bigotimes_{k=1}^{n-1} \sigma_{k}^{z}\right) \otimes\left(\sigma_{n}^{x}+\mathrm{i} \sigma_{n}^{y}\right),
$$

and the $2 N$ Majorana fermions

$$
\check{\gamma}_{2 n}=\frac{1}{\mathrm{i} \sqrt{2}}\left(\hat{a}_{n}-\hat{a}_{n}^{\dagger}\right), \quad \check{\gamma}_{2 n-1}=\frac{1}{\sqrt{2}}\left(\hat{a}_{n}+\hat{a}_{n}^{\dagger}\right) .
$$

These fulfill $\left\{\check{\gamma}_{m}, \check{\gamma}_{n}\right\}=\delta_{m n}$, and are delocalized in terms of the original lattice of fermions. The Majorana fermions diagonalize the Hamiltonian in the sense that

$$
H=\sum_{m n} C_{m n} \check{\gamma}_{m} \check{\gamma}_{n}
$$

with an Hermitian $2 N \times 2 N$ matrix $C$. Next, define the imaginary and anti symmetric correlation matrix $\Gamma_{i j}=\left\langle\left[\check{\gamma}_{i}, \check{\gamma}_{j}\right]\right\rangle$. We will consider only the ground state, and thus the expectation values are to be taken in the ground state. In this sense, our model is defined by the matrix $C$ while the state is defined in the matrix $\Gamma$. The Majorana fermions have a two-to-one correspondence to the fermions (4), and hence tracing out a particle from the system amounts to removing the two adjacent rows and columns in $\Gamma$ corresponding to the particle. Tracing out $N-N^{\prime}$ particles this way, we end up with the $2 N^{\prime} \times 2 N^{\prime}$ matrix $\bar{\Gamma}_{i j}$. This latter matrix can be made block diagonal through an orthogonal transformation $O$, such that we can define new Majorana fermions $\bar{\gamma}_{n}$ that fulfill

$$
O^{\mathrm{T}} \bar{\Gamma} O=\left\{\left\langle\left[\bar{\gamma}_{i}, \bar{\gamma}_{j}\right]\right\rangle\right\}=\bigoplus_{k=1}^{N^{\prime}}\left(\begin{array}{cc}
0 & \mathrm{i} \xi_{k} \\
-\mathrm{i} \xi_{k} & 0
\end{array}\right)
$$

with $0 \leq \xi_{k} \leq 1$. The transformation $O$ is the same transformation that block diagonalizes the Hamiltonian matrix $C$. The matrix $\bar{\Gamma}$ corresponds to the state described by the reduced density matrix $\rho^{\prime}$, with the $N-N^{\prime}$ particles traced out. The fermion operators $\bar{a}_{n}$ corresponding to $\bar{\gamma}_{n}$ will diagonalize the reduced density matrix such that the eigenvalues thereof can be computed. The eigenvalues are determined by the set of binary occupation numbers $\eta=\left\{n_{k}\right\}, k=1,2, \ldots, N^{\prime}$, and $n_{k}=\{0,1\},|\eta\rangle$ being an eigenstate of $\bar{a}_{k} \bar{a}_{k}^{\dagger}$ with eigenvalue $n_{k}$. Thus $|\eta\rangle$ is also an eigenstate of $\rho^{\prime}$ and the reduced density matrix is $\rho^{\prime}=\sum_{\eta} \lambda_{\eta}|\eta\rangle\langle\eta|$ with eigenvalues

$$
\lambda_{\eta}=\prod_{k=1}^{N^{\prime}}\left[\frac{1}{2}+(-1)^{n_{k}} \xi_{k}\right] .
$$

Finally, the entropy becomes

$$
S_{\mathscr{A}}=\sum_{k=1}^{N} H\left(\frac{1}{2}\left(1+\xi_{k}\right)\right),
$$

where $H(x)=-x \log _{2} x-(1-x) \log _{2}(1-x)$ is the binary entropy function.

The entropy of the ground state as measured by Eq. (2) when the system is partitioned into two equal parts, is shown in Fig. 1. The increased entropy around the criti-

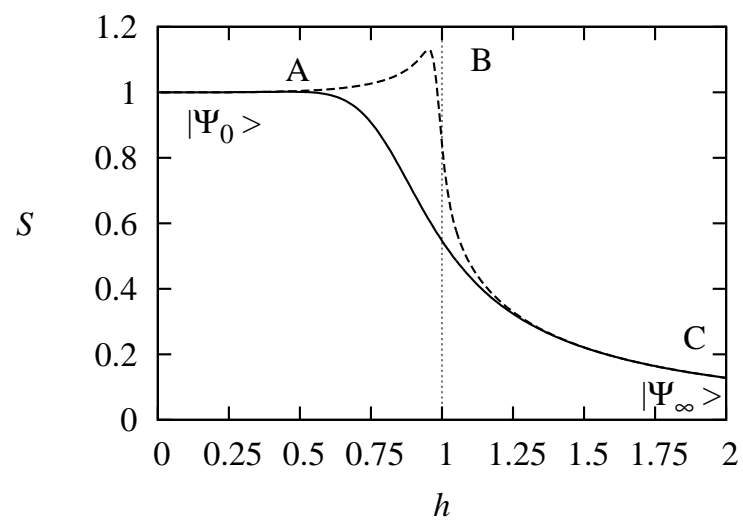

FIG. 1: The entropy in the quantum Ising model as function of the external magnetic field $h$ for $N=10$ (lower line) and $N=100$ particles. In both cases half system is traced out, from the edge. The letters refer to the places of investigation in Fig. 2] The critical point (in the thermodynamic limit) is shown as a vertical line.

cal point is a hallmark of the quantum phase transition, though this does not show in the small system.

Figure 2 shows the magnitude of the decreasingly ordered eigenvalues $\lambda_{n}^{\downarrow}$. Note that there are $2^{N / 2}=128$ possible eigenvalue contributions, while this shows that only a very few of these contribute to the wave function, and thus to the entanglement as measured by the entropy $S$.

The main eigenvalues at critical point decrease roughly exponentially, as $\lambda_{n}^{\downarrow} \sim e^{-2 n}$, while off criticality only the very first contribute significantly. In the low field limit, the highest eigenvalues are paired due to the Schrödinger cat nature of the state, while in the high field limit there is one single main contribution, which is the product state $\left|\Psi_{\infty}\right\rangle$. With PBC, the eigenvalues would be paired also 


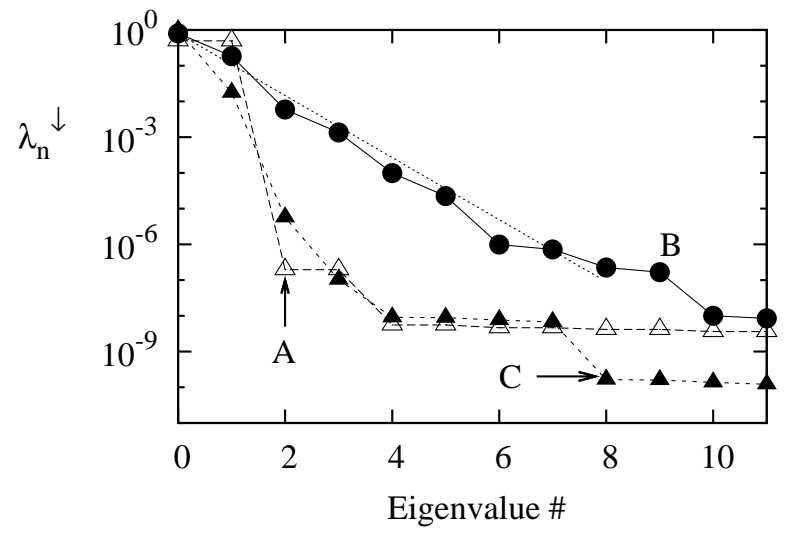

FIG. 2: The decreasingly ordered eigenvalues $\lambda_{n}^{\downarrow}$ of the reduced density matrix of the Ising model's ground state when tracing out half size. Data for $N=50$. The upper line $(\bullet)$ denotes the critical values when $h=h_{c}=1$, at (B) in figure 1. $(\triangle)$ denotes the Scrödinger cat state with $h=0.5$, at (A); and ( $\boldsymbol{\Delta})$ denotes the approximate product state with $h=2$, at (C). The fitting line to the critical values $\lambda_{n}^{\downarrow} \sim e^{-2 n}$ is shown. Only the 10 largest eigenvalues are shown since numerical errors become prominent after this.

for $h>1$, except for the single main eigenvalue due to the translational symmetry. This pairing also occurs in a conformal bosonic chain [10], but is not a general property of a non critical system.

Hence we can approximate the wave function by restricting the sum (1) to some upper limit $\chi^{\prime}<\chi$ to make the new wave function

$$
\left|\Psi^{\prime}\right\rangle=\sum_{n=1}^{\chi^{\prime}} \sqrt{\lambda_{n}^{\prime}}\left|\psi_{n}^{(\mathscr{A})}\right\rangle \otimes\left|\psi_{n}^{(\mathscr{B})}\right\rangle
$$

where the new coefficients $\lambda_{n}^{\prime}$ are determined by normalization, $\lambda_{n}^{\prime}=\lambda_{n} /(1-\varepsilon)$. We define an error as

$$
\varepsilon=\sum_{n=\chi^{\prime}+1}^{\chi} \lambda_{n}
$$

which measures the difference in the eigenvalue sum of the two wave functions. The overlap becomes

$$
\left\langle\Psi^{\prime} \mid \Psi\right\rangle=\sqrt{1-\varepsilon} .
$$

\section{SCALING OF EIGENVALUES}

We investigate how the eigenvalues scale with increasing system size. Off the critical point there is no scaling with system size of the eigenvalues since the entropy saturates at some value. However, on, or near the critical point the entanglement entropy diverges as predicted by conformal field theory. Given that we trace out a constant fraction of the entire system, the entanglement entropy diverges up to an additive constant as $[6,17]$

$$
S_{\mathscr{A}} \sim \frac{c}{6} \log _{2}(N)
$$

where $c$ is the central charge of the conformal field theory corresponding to the phase transition. In the Ising case $c=1 / 2$.

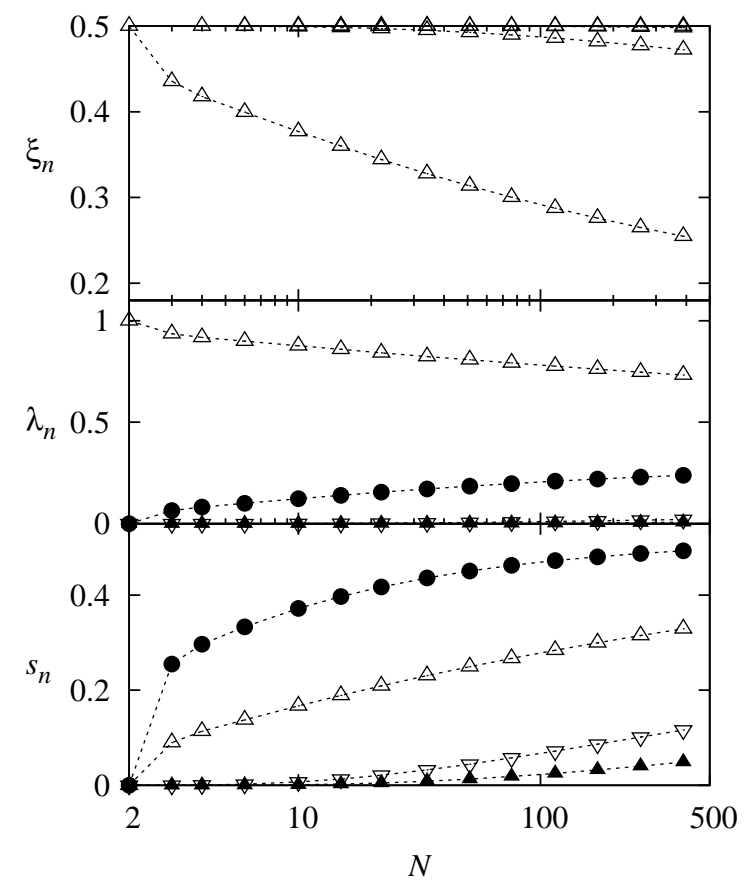

FIG. 3: From the top, the figures show the four smallest eigenvalues of the correlation matrix in Eq. (5), the four largest eigenvalues of the density matrix as computed from Eq. (6), and the corresponding entropy contributions $s_{n}=$ $-\lambda_{n} \log \lambda_{n}$. For the two lower graphs, equal point types refer to the same eigenvalues. System is at the critical Ising point and is traced at half size.

Figure 3 shows how the largest eigenvalues scale at the critical Ising point. The largest eigenvalue $\lambda_{1}^{\downarrow}(N) d e$ creases with system size at criticality, while the other increases. The largest decrease roughly as $\lambda_{1}^{\downarrow}(N) \sim$ $-0.027 \log N$, while the second increases roughly as $\lambda_{2}^{\downarrow}(N) \sim 0.021 \log N$, both within the range shown in Fig. 3. However, all entropy contributions increase with $N$, thus contributing to the overall divergence of the entanglement entropy.

\section{APPROXIMATING THE GROUND STATE}

There exists successful techniques to approximate the ground state of a quantum system, the most prominent of which is the DMRG scheme 13. Rather than taking only ground states of a sub lattice when renormalizing the system, this method takes into account possible entangled states as well, producing unprecedented accuracy. The role of entanglement in DMRG is still under investigation, but it seems clear that it is vital to the success of the scheme 14]. The results of our work here are indicative that the main contributions to the ground state 
are indeed entangled at the critical point, along with few of these terms contributing to the actual wave function. These results are not directly applicable to an improvement of the DMRG algorithm, but rather indications as to the success of DMRG. Furthermore, any prospective technique to find the ground state of a quantum system, even in those areas where known techniques fail, needs to understand the nature of the wave function in the system, and we believe that the entanglement properties would play an important part in such a method. In particular, the entanglement properties is precisely what distinguishes a quantum many-body system from the classical counterpart, and entanglement must therefore be an essential part of any such method.

Having found that only few terms contribute to the entanglement in the ground state, we query how well we could possibly approximate the ground state of the full system by the first few terms in the Schmidt decomposition. To this end, we focus on the few-particle case, where the exact wave functions can be computed explicitly. We split the open spin chain in two partitions, and compute the overlap between the actual wave function and the Schmidt expansion of the two subsystems, ordered decreasingly on those terms with the largest overlaps.

$$
\mathcal{O}_{n}=\left\langle\Psi \mid \psi_{n}^{(\mathscr{A})}\right\rangle \otimes\left|\psi_{n}^{(\mathscr{B})}\right\rangle .
$$

A priori it is clear that in the zero field limit $\mathcal{O}_{1}=\mathcal{O}_{2}=$ $1 / \sqrt{2}$, while any higher terms vanish. In the high-field limit, $\mathcal{O}_{1}=1$ as the only non vanishing overlap. We assume that the wave functions $\left|\psi_{n}^{(\cdot)}\right\rangle$ are ordered with decreasing Schmidt number, and the results are shown in Fig. 4

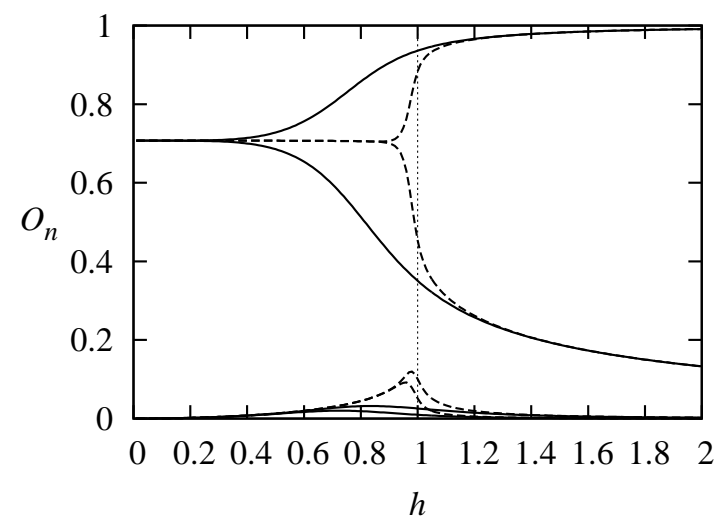

FIG. 4: The four largest overlaps $\mathcal{O}_{n}, n=1,2,3,4$ (from top to bottom) with magnetic field $h$ and system sizes $N=10$ (full lines) and $N=100$ (dashed lines). Again, the critical point is indicated with the vertial line.

Since the fermions $\bar{a}_{k}$ corresponding to the Majorana fermions defined in Eq. (5) diagonalize the reduced density matrix, the overlap follows straightforwardly. The probability of the state $|\eta\rangle$ given the density matrix $\rho^{\prime}=\lambda_{\eta}|\eta\rangle\langle\eta|$ is $\lambda_{\eta}$, and the overlap between this and

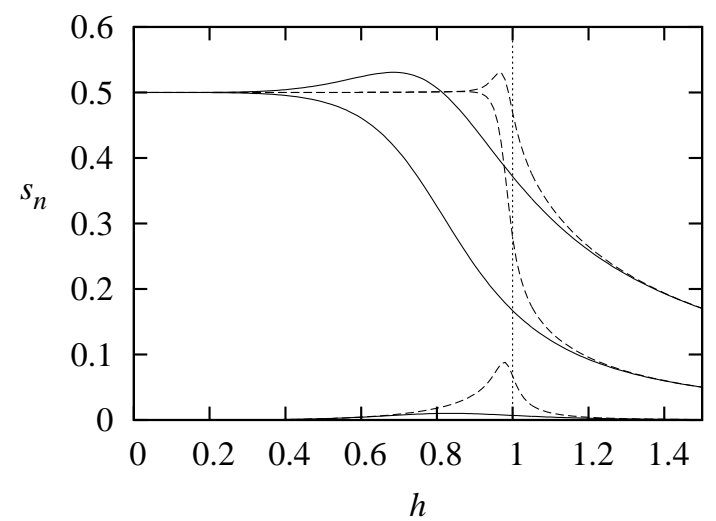

FIG. 5: The three main entropy contributions $s_{n}, n=1,2,3$ (from top to bottom) in the Ising model with magnetic field $h$. Full lines are for $N=10$, while dashed lines are for $N=100$.

the ground state will therefore be $\sqrt{\lambda_{\eta}}$, or

$$
\mathcal{O}_{\eta}=\prod_{k} \sqrt{\frac{1}{2}+(-1)^{n_{k}} \xi_{k}}
$$

Most prominently, the $\mathcal{O}_{1}$ overlap increases monotonically as $h$ increases. That is, even at criticality this first term consisting of the ground states at each side approximates the actual wave function even better that it does for the cat-state where the overlap naturally is $1 / \sqrt{2}$. The increased entropy at critical points is mainly due to other terms rising around criticality, terms that are not part of the entire wave function at non critical points in the phase space.

Investigating the Schmidt coefficients' entropy contributions, the results are shown in Fig. 5 Hence we see that apart from the two major contributions that are equally prominent at $h \rightarrow 0$, there is one more contributing at criticality. The effect is mainly pronounced with big systems, but a trace is also seen at $N=10$. The increased entropy at the critical point is contributed to by the largest term, but also by the appearance of terms that are zero at all noncritical points.

The error in the overlap and in the entropy that arises from only choosing $\chi^{\prime}<\chi$ terms in the Schmidt decomposition is zero (or very close) off criticality, since the Schmidt rank is very small. However, on criticality $\chi$ in principle extends to the dimension of the Hilbert space. However, the errors in the overlap $\delta \mathcal{O}=1-\sum_{n=1}^{\chi^{\prime}} \mathcal{O}_{n}$ and entropy $\delta S=S-\sum_{n=1}^{\chi^{\prime}} s_{n}$ are plotted in Fig [6] We see that the errors grow linearly with $N$ when $N>100$. The number of Schmidt terms needed to compute the entropy up to an error of $10^{-4}$, or the effective Schmidt number is only 4 up to a system of $N=400$ sites. 


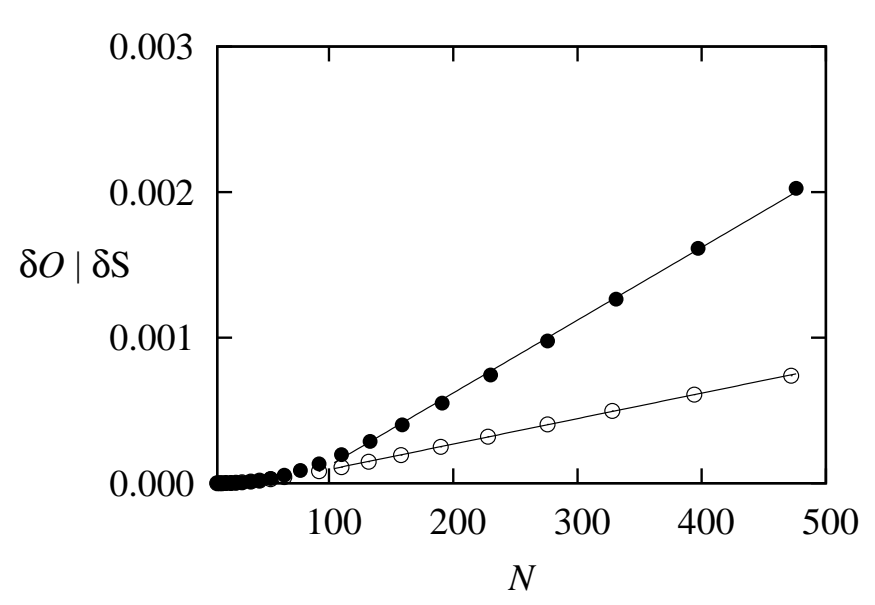

FIG. 6: The errors $\delta \mathcal{O}(\circ)$ and $\delta S(\bullet)$ as defined in the text with different system sizes at the critical Ising point. For $\delta \mathcal{O}$ we have chosen $\chi^{\prime}=4$ and for $\delta S \chi^{\prime}=3$. In all cases the system is traced at half-size. The lines for $N>100$ are linears $\delta \mathcal{O} \simeq 5 \times 10^{-6} N-3.8 \times 10^{-4}$ and $\delta S \simeq 1.75 \times 10^{-6} N-8.1 \times$ $10^{-5}$. For small systems the linearity does not hold.

\section{Conclusions}

We have seen that the complex structure of a wave function at critical point in the Ising model comes from the emergence of several terms in the Schmidt decomposition of the wave function. Nevertheless, remarkably few terms actually contribute to the expansion, and physical properties such as the entropy can be extracted using very few terms, even at criticality. Mainly, the rapid decrease of the Schmidt coefficients on or off criticality identifies this effect. We have detailed the effects with both large and small systems, and the primary conclusions hold in both cases.

The matter that these few eigenstates contribute to physical properties such as the entropy and also the overlap with the true ground state signifies the fact that finding the true ground state, or an approximation to it, is not a complicated task per se, we known that only a few of the eigenstates of the Hamiltonian would suffice for a sufficiently good description of the state. The results in this paper also indicate that these states are indeed entangled, and thus any algorithm that does not include entanglement is bound to fail.

The effective Schmidt number that is needed to find a state within some limit is small, on the order of 10 even up to several hundred particles. Thus the effective Hilbert space is dramatically reduced. However, we have not addressed the problem of determining the unknown ground state of a system, only pointed out that the problem is not consistently hard given the correct (so far unknown) approach.

\section{Acknowledgments}

The author is greatly indebted to Kåre Olaussen for valuable contributions to this work. Susanne Viefers and the NordForsk network on low-dimensional physics is thanked for hosting valuable meetings. The University of Troms $\varnothing$ (UiT $\varnothing$ ) is thanked for providing office space.
[1] A. Einstein, B. Podolsky, and N. Rosen, Phys. Rev. 47, 777 (1935).

[2] J. S. Bell, Physics 1, 195 (1964).

[3] M. A. Nielsen and I. L. Chuang, Quantum Computation and Quantum Information (Cambridge University Press, Cambridge, UK, 2000).

[4] S. Sachdev, Quantum Phase Transitions (Cambridge University Press, Cambridge, UK, 1999).

[5] A. Osterloh, L. Amico, G. Falci, and R. Fazio, Nature 416, 608 (2002).

[6] G. Vidal, J. I. Latorre, E. Rico, and A. Kitaev, Phys. Rev. Lett. 90, 227902 (2002).

[7] S. O. Skrøvseth and K. Olaussen, Phys. Rev. A 72, 022318 (2005).

[8] T. J. Osborne and M. A. Nielsen, Phys. Rev. A 66, 032110 (2002).
[9] V. E. Korepin, Phys. Rev. Lett. 92, 096402 (2004).

[10] S. O. Skrøvseth, Phys. Rev. A 72, 062305 (2005).

[11] P. Calabrese and J. Cardy, J. Stat. Mech.: Theory Exp. (2005) P04010.

[12] J. I. Latorre, C. A. Lütken, E. Rico, and G. Vidal, Phys. Rev. A 71, 034301 (2005).

[13] S. R. White, Phys. Rev. Lett. 69, 2863 (1992).

[14] U. Schollwöck, Rev. Mod. Phys. 77, 259 (2005).

[15] G. Vidal (2005), cond-mat/0512165.

[16] P. D. Francesco, P. Mathieu, and D. Sénéchal, Conformal Field Theory (Springer, New York, 1997).

[17] P. Calabrese and J. Cardy, J. Stat. Mech.: Theory Exp (2004) P06002.

[18] J. I. Latorre, E. Rico, and G. Vidal, Quant. Inf. and Comp. 4, 48 (2004), quant-ph/0304098. 